\title{
MR/PET or PET/MRI: does it matter?
}

\author{
Thomas Beyer $\cdot$ Ewald Moser
}

Received: 22 November 2012/Revised: 20 December 2012/Accepted: 21 December 2012/Published online: 6 February 2013 (C) ESMRMB 2013

\begin{abstract}
After the very successful clinical introduction of combined PET/CT imaging a decade ago, a hardware combination of PET and MR is following suit. Today, three different approaches towards integrated PET/MR have been proposed: (1) a triple-modality system with a 3T MRI and a time-of-flight PET/CT installed in adjacent rooms, (2) a tandem system with a 3T MRI and a time-of-flight $\mathrm{PET} / \mathrm{CT}$ in a co-planar installation with a joint patient handling system, and (3) a fully-integrated system with a whole-body PET system mounted inside a 3T MRI system. This special issue of MAGMA brings together contributions from key experts in the field of PET/MR, PET/CT and $\mathrm{CT}$. The various papers share the author's perspectives on the state-of-the-art PET/MR imaging with any of the three approaches mentioned above. In addition to several reviews discussing advantages and challenges of combining PET and MRI for clinical diagnostics, first clinical data are also presented. We expect this special issue to nurture future improvements in hardware, clinical protocols, and efficient post-processing strategies to further assess the diagnostic value of combined PET/MR imaging. It remains to be seen whether a so-called "killer application" for PET/ MRI will surface. In that case PET/MR is likely to excel in
\end{abstract}

Thomas Beyer and Ewald Moser contributed equally to this article.

T. Beyer $(\square)$

cmi-experts GmbH, Wannenholzstr 26, 8046 Zurich,

Switzerland

e-mail: thomas.beyer@cmi-experts.com

\section{E. Moser}

MR Center of Excellence, Center for Medical Physics and

Biomedical Engineering, Medical University of Vienna,

Lazarettgasse 14, Vienna, Austria

e-mail: ewald.moser@meduniwien.ac.at pre-clinical and selected research applications for now. This special issue helps the readers to stay on track of this exciting development.

Keywords PET/MR - PET/CT - Attenuation correction * Artifacts $\cdot$ Safety $\cdot$ Cancer

Driven by the fast-pace advances of combined imaging, editors and authors deemed it timely to provide the readers of MAGMA with a state-of-the-art summary of the status of combined PET and MR imaging. Or shall we say, combined MR and PET imaging? Or rather, PET/MR, or MR/PET? Does it matter?

Well, it does to the heart of most of the readers. Depending on their scientific and clinical background, people would call this new imaging kid on the block either $\mathrm{PET} / \mathrm{MR}$, in case their origin is in the PET community, or $\mathrm{MR} / \mathrm{PET}$, if they are more sympathetic to the field of diagnostic radiology. Either way, what's in a name? Time and linguists will tell.

However, the introduction of a new combined and even fully integrated PET and MR imaging system should give rise to a clear separation (and subsequently definition) of retrospective and prospective image fusion. In this special issue we propose to use the term PET/MRI (or MR/PET) spelled with a backslash - in case of hardware-based integration of PET and MRI into a single gantry system. In the case of retrospective image alignment, based on two separate imaging systems, we advocate the use of PET-MR, or MR-PET-spelled with a hyphen. This attempt to clearly denote the origin of the fused images should give credit to the origins of the combined image information and, subsequently, to the challenges of either approach to multimodality imaging. 
Unlike PET/CT (note, very few people call it CT/PET, which could be another argument in favour of PET/MR), PET/MR did start out in the pre-clinical arena before being promoted to the clinical arena [1], together with significant industry funding, at a time when no clear clinical indication was defined [2, 3]. Even today, seven years into the use of PET/MR in humans, no definite indication has been determined for clinical use cases. This is ever more surprising because the integrated hardware design is expensive and healthcare costs are under scrutiny [4]. Nonetheless, numerous institutions invest into this new imaging combination, hoping that a so called "killer application" for PET/MR is found either in-house, by a friend who also has access to such machinery, or elsewhere.

This special issue brings together several perspectives on the state-of-the-art of PET/MR imaging. The series of adjoined manuscripts opens with a contribution by Kalemis and co-authors from Philips, who summarize a manufacturer's perspective on the first commercial system that provides whole-body PET/MR images [5]. Of note, we also invited other major suppliers of hardware combinations to contribute equally in separate articles, however, without a positive response.

This article is followed by a contribution from VeitHaibach et al. [6] from Zurich, who took delivery of an alternative, triple-modality PET/CT-MR system over a year ago. This system combines a whole-body PET/CT and a 3T MRI in adjacent rooms that are linked with a shuttle system. Major advantages are the ability to work with two established, unaltered imaging modalities (PET/CT and $\mathrm{MRI}$ ) and the ability to perform CT-based attenuation correction of the PET data [7], which is a pre-requisite for fully quantitative non-invasive metabolic information. The triple-modality system has also been argued to be the most cost-effective approach towards combined imaging [8].

The third paper is a contribution by Kjær et al. [9] from Copenhagen, who summarize their experiences with the third design concept being the most integrated PET/MR system, based on a new PET detector design that is inserted completely into a 3T whole-body MRI system. The authors elaborate on clinical use case scenarios for fully integrated imaging. This leads to more clinical contributions, by Platzek et al. [10] from Dresden, who present first data from sequential PET/MR imaging for therapy response assessment in malignant lymphoma. Clearly, with the yet limited availability of PET/MR, the complexity of defining imaging protocols and the overall slow rise of clinical studies, data like these are helpful and needed in assessing the clinical potential of this imaging modality [11-13].

Vargas et al. [14] from Geneva address the complexity of defining optimum imaging protocols fit for clinical application using a sequential PET/MR design [5]. The message of their report is that a priori multi-disciplinary efforts are needed for the timely adoption of standardized imaging protocols for clinical studies.

We also invited Cho et al. [15] from Gachon University in Korea to share with the readers their experience with an affiliated PET-MRI system, that is the side-by-side arrangement of a 7T MRI and a brain-only high-resolution research brain tomograph (HHRT) [16]. While this set-up is unique and not commercially available, it does offer insight into where PET-MRI could go in the future and sets the stage for considering advancing on the technological platform if there are well-defined clinical needs or research requirements.

While the introduction of PET/CT was supported by the need to provide anato-metabolic imaging [17] in clinical oncology, the same cannot be said for the adoption of PET/ MR just now. Furthermore, a number of additional methodological challenges are yet to be resolved. Keereman and colleagues from Ghent summarize these in their contribution to this special issue [18]. The general challenge is (not limited to) the need to derive CT-like attenuation factors from the available MR images. Wagenknecht et al., from Jülich further discuss this [19] by describing in detail various approaches to MR-based attenuation (and scatter) correction (MR-AC) [20] and provide a summary of the different methods that are available today. Finally, on the note of quantitative accuracy, Schramm et al., from Dresden compare the accuracy of MR-based attenuation correction in clinical patients to the gold standard, which is PET transmission imaging using a positron-emitting transmission source [21]. This data is unique in that such comparisons are lacking in clinical scenarios due to the shortage of PET-only imaging systems that employ PETtransmission source imaging.

Having discussed current approaches to MR-AC, Larsson and colleagues from Umeå present a new approach to deriving MR-based substitute CT images for the purpose of attenuation correction of emission images of the head [22]. These substitute CT images include information on bone structures that contribute significantly to the overall attenuation of the emission data. First results are rather convincing and the reader is encouraged to stay put for further reports on this approach.

The latter part of this special issue is dedicated to further important contributions. Pietrzyk and Herzog from Jülich [23] reflect on the term "co-registration" in the context of PET/MR imaging, both, in terms of spatial and temporal alignment; an interesting read to anybody in search of an in-depth perspective regarding the message of "fully integrated PET and MR information". Further, Gillings from Copenhagen [24] sheds light on the biochemical perspective of the molecular imaging tracer used for PET, particular in combination with MRI. This contribution, aimed 
specifically at the readers of MAGMA, provides insight into the choices of PET tracer molecules and the noninvasive imaging and quantification of pre-selected biochemical processes that could benefit from complementary MR information.

Having discussed at length the pros and cons of PET/ $\mathrm{MR}$, and MR/PET, the reader is still left in the dark about the requirements for the actual installation of such a combined imaging system. Sattler and colleagues from Leipzig relieve the readers from this frustration [25]. Their contribution summarizes the physical and organizational provisions for the installation and operation of a PET/MR system.

Finally, having been able to install such an imaging machine, turning it on, assuming that all protocols have been optimized and clinical studies are lined up, the first images may, however, still look strange to the eyes of the operator. Keller et al., from Copenhagen provide a closer look at the most common image artifacts observed in clinical operations, which can help the reader (and operator) decide whether their first images are the best to be achieved with the imaging system, or further optimization and corrections are required [26]. Artifacts matter, particularly when adopting novel imaging techniques, and may be a stimulating source for future improvements.

Here we would like to add a note on the safety of this novel combined imaging approach [27]. Although legal safety aspects are well understood for either imaging modalities alone, safety regulations are rather different. In PET imaging, safety of the patients and staff relate mainly to the handling of the tracer and subsequent radiation safety measures for the ionizing, high-energy $(511 \mathrm{keV})$ radiation. In contrast, in MRI, ionizing radiation is not a subject of concern; however, static magnetic fields may potentially lead to ballistic effects, fast gradients may cause peripheral nerve stimulation, and radio-frequency irradiation could translate into increased specific absorption rate and, potentially, tissue heating [28, 29]. Imaging procedures and safety measures grow in complexity when moving PET and MR physically and methodologically closer together. This needs to be addressed in advanced training of the staff involved in combined PET/MR imaging procedures.

It is the result of human creativity that two imaging methods so different in their physics background and so alien in their technical requirements have been merged sucsessfully through scientific dedication and technical innovation.

Does it matter, whether we call it PET/MR or MR/PET? Probably not. And not as much as we need to define which clinically relevant information each component shall contribute to the combined data. Integrating images requires also the integration of professionals, including combined training efforts as well as a close and mutually beneficial collaboration. An optimum clinical interpretation of the combined data clearly requires a new mindset and a vision. Let's get to work.

Acknowledgments We like to thank Marguerite Izquierdo from the MAGMA Editorial office for her continuous and courteous support and professional advice during the preparation of this special issue.

\section{References}

1. Wehrl HF, Judenhofer MS, Wiehr S, Pichler BJ (2009) Preclinical PET/MR: technological advances and new perspectives in biomedical research. Eur J Nucl Med Mol Imaging 36(Suppl 1):S56-S68

2. Pichler BJ, Kolb A, Nägele T, Schlemmer HP (2010) PET/MRI: paving the way for the next generation of clinical multimodality imaging applications. J Nucl Med 51(3):333-336

3. Beyer T, Freudenberg LS, Czernin J, Townsend DW (2011) The future of hybrid imaging-part 3: PET/MR, small-animal imaging and beyond. Insights Imaging 2(3):235-246

4. Goyen M, Debatin JF (2009) Healthcare costs for new technologies. Eur J Nucl Med Mol Imaging 36(Suppl 1):S139-S143

5. Kalemis A, Delattre BM, Heinzer S (2013) Sequential wholebody PET/MR scanner: concept, clinical use, and optimisation after two years in the clinic. The manufacturer's perspective. Magn Reson Mater Phy 26(1):5-23

6. Veit-Haibach P, Kuhn FP, Wiesinger F, Delso G, von Schulthess G (2013) PET-MR imaging using a tri-modality PET/CT-MR system with a dedicated shuttle in clinical routine. Magn Reson Mater Phy 26(1):25-35

7. Kinahan PE, Townsend DW, Beyer T, Sashin D (1998) Attenuation correction for a combined 3D PET/CT scanner. Med Phys 25(10):2046-2053

8. von Schulthess GK, Burger C (2010) Integrating imaging modalities: what makes sense from a workflow perspective? Eur J Nucl Med Mol Imaging 37(5):980-990

9. Kjær A, Loft A, Law I, Berthelsen A, Borgwardt L, Löfgren J, Johnbeck C, Hansen A, Keller S, Holm S, Højgaard L (2013) PET/MRI in cancer patients: first experiences and vision from Copenhagen. Magn Reson Mater Phy 26(1):37-47

10. Platzek I, Beuthien-Baumann B, Langner J, Popp M, Schramm G, Ordemann R, Laniado M, Kotzerke J, van den Hoff J (2013) PET/ MR for therapy response evaluation in malignant lymphoma: initial experience. Magn Reson Mater Phy 26(1):49-55

11. Antoch G, Bockisch A (2009) Combined PET/MRI: a new dimension in whole-body oncology imaging? Eur J Nucl Med Mol Imaging 36(Suppl 1):S113-S120

12. Buchbender C, Heusner TA, Lauenstein TC, Bockisch A, Antoch G (2012) Oncologic PET/MRI, part 1: tumors of the brain, head and neck, chest, abdomen, and pelvis. J Nucl Med 53(6):928-938

13. Buchbender C, Heusner TA, Lauenstein TC, Bockisch A, Antoch G (2012) Oncologic PET/MRI, part 2: bone tumors, soft-tissue tumors, melanoma, and lymphoma. J Nucl Med 53(8):1244-1252

14. Vargas MI, Becker M, Garibotto V, Heinzer S, Loubeyre P, Gariani J, Lovblad K, Vallée JP, Ratib O (2013) Approaches for the optimization of MR protocols in clinical hybrid PET/MRI studies. Magn Reson Mater Phy 26(1):57-69

15. Cho ZH, Son YD, Choi EJ, Kim HK, Kim JH, Lee SY, Ogawa S, Kim YB (2013) In-vivo human brain molecular imaging with a brain-dedicated PET/MRI system. Magn Reson Mater Phy 26(1):71-79

16. van Velden FH, Kloet RW, van Berckel BN, Buijs FL, Luurtsema G, Lammertsma AA, Boellaard R (2009) HRRT versus HR+ 
human brain PET studies: an inter scanner test-retest study. J Nucl Med 50(5):693-702

17. Wahl RL, Quint LE, Cieslak RD, Aisen AM, Koeppe RA, Meyer CR (1993) "Anatometabolic" tumor imaging: fusion of FDG PET with CT or MRI to localize foci of increased activity. J Nucl Med 34(7):1190-1197

18. Keereman V, Mollet P, Berker Y, Schulz V, Vandenberghe S (2013) Challenges and current methods for attenuation correction in PET/MR. Magn Reson Mater Phy 26(1):81-98

19. Wagenknecht G, Kaiser HJ, Mottaghy FM, Herzog H (2013) MRI for attenuation correction in PET: methods and challenges. Magn Reson Mater Phy 26(1):99-113

20. Hofmann M, Pichler B, Schölkopf B, Beyer T (2009) Towards quantitative PET/MRI: a review of MR-based attenuation correction techniques. Eur $\mathrm{J}$ Nucl Med Mol Imaging 36(Suppl 1):S93-S104

21. Schramm G, Langner J, Hofheinz F, Petr J, Beuthien-Baumann B, Platzek I, Steinbach J, Kotzerke J, van den Hoff J (2013) Quantitative accuracy of attenuation correction in the Philips Ingenuity TF whole-body PET/MR system: a direct comparison with transmission-based attenuation correction. Magn Reson Mater Phy 26(1):115-126

22. Larsson A, Johansson A, Axelsson J, Nyholm T, Asklund T, Riklund K, Karlsson M (2013) Evaluation of an attenuation correction method for PET/MR imaging of the head based on substitute CT images. Magn Reson Mater Phy 26(1):127-136
23. Pietrzyk U, Herzog H (2013) Does PET/MR in human brain imaging provide optimal registration? A critical reflection. Magn Reson Mater Phy 26(1):137-147

24. Gillings N (2013) Radiotracers for positron emission tomography imaging. Magn Reson Mater Phy 26(1):149-158

25. Sattler B, Jochimsen T, Barthel H, Sommerfeld K, Stumpp P, Hoffmann KT, Gutberlet M, Villringer A, Kahn T, Sabri O (2013) Physical and organizational provision for installation, regulatory requirements and implementation of a simultaneous hybrid PET/MR-imaging system in an integrated research and clinical setting. Magn Reson Mater Phy 26(1):159-171

26. Keller SH, Holm S, Hansen AE, Sattler B, Andersen F, Klausen TL, Højgaard L, Kjær A, Beyer T (2013) Image artifacts from MR-based attenuation correction in clinical, whole-body PET/ MRI. Magn Reson Mater Phy 26(1):173-181

27. Brix G, Nekolla EA, Nosske D, Griebel J (2009) Risks and safety aspects related to PET/MR examinations. Eur J Nucl Med Mol Imaging 36(Suppl 1):S131-S138

28. Moser E, Stadlbauer A, Windischberger C, Quick HH, Ladd ME (2009) Magnetic resonance imaging methodology. Eur J Nucl Med Mol Imaging 36(Suppl 1):S30-S41

29. Moser E, Stahlberg F, Ladd ME, Trattnig S (2012) 7-T MRfrom research to clinical applications? NMR Biomed 25(5): $695-716$ 